\title{
Stents autoexpandibles biodegradables en endoscopia intervencionista gastrointestinal y pancreatobiliar. Estado actual
}

\section{The Current State of Biodegradable Self-expanding Stents in Interventional Gastrointestinal and Pancreatobiliary Endoscopy}

Óscar A. Álvarez B., MD, ${ }^{1}$ Rodrigo Castaño Llano, MD, ${ }^{2}$ David Restrepo. ${ }^{3}$

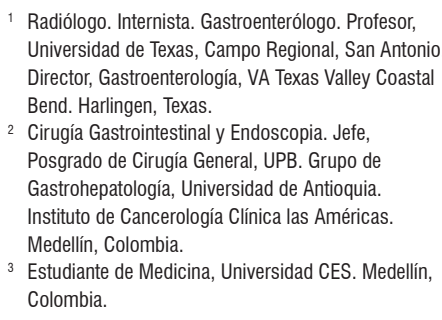

${ }^{3}$ Estudiante de Medicina, Universidad CES. Medellín, Colombia.

Fecha recibido: $08-07-14$ Fecha aceptado: 06-04-15

\begin{abstract}
Resumen
Los stents biodegradables son una opción muy atractiva para utilizar en pacientes con estenosis benignas recurrentes/recalcitrantes del tracto digestivo y biliar. En teoría, utilizando estos stents biodegradables, se pueden evitar dilataciones repetitivas de estenosis digestivas o biliares refractarias al manejo convencional, como también se podrían evitar resecciones quirúrgicas, principalmente en pacientes de alto riesgo quirúrgico, y se minimiza el número de procedimientos endoscópicos intervencionistas.
\end{abstract}

\section{Palabras clave}

Stent reabsorbible, estenosis biliares benignas, estenosis colónicas benignas, estenosis gastroduodenales benignas.

\begin{abstract}
Biodegradable stents are a very attractive option for use in patients with benign but recurrent and recalcitrant digestive tract and biliary strictures. In theory, use of these biodegradable stents mitigates the need for repetitive expansion of digestive or biliary strictures which are refractory to conventional management and avoids the need of surgical resection. This is especially true for patients at high surgical risk. Stents can also minimize the number of interventional endoscopic procedures performed on a patient.
\end{abstract}

Keywords

Reabsorbable stent, benign biliary stenoses, benign colon stenoses, benign gastroduodenal stenoses.

\section{INTRODUCCIÓN}

El uso de stents metálicos autoexpandibles ha demostrado ser un tratamiento efectivo para manejar las estenosis benignas refractarias y malignas del tracto digestivo. Sin embargo, el uso de estos stents, principalmente en el escenario de las patologías benignas, está asociado con varios tipos de complicaciones como son las dificultades al momento de la extracción, la migración y la hiperplasia y también está relacionado con los costos inherentes de procedimientos endoscópicos repetitivos (1-3). Los stents plásticos se aso- cian con una mayor tasa de migración, una menor flexibilidad y una menor fuerza radial expansiva $(4,5)$.

En las últimas dos décadas se han hecho avances muy significativos en el desarrollo de materiales biocompatibles y biodegradables para la aplicación médica. Es así como se han logrado desarrollar los stents biodegradables con el fin de intentar remediar los problemas ya mencionados (6). El presente artículo revisa la experiencia acumulada hasta la fecha, que no es mucha y es poco conocida, y se hace énfasis en los aspectos biomecánicos con la intención de considerar una futura implementación local. 


\section{BIOMATERIALES}

Un biomaterial es un compuesto inerte diseñado para ser implantado o incorporado dentro de un sistema biológico vivo. Los biomateriales son sometidos a situaciones adversas, dado que están expuestos de modo temporal o permanente a fluidos del cuerpo, donde se da la corrosión de los componentes del implante. Los biomateriales pueden restituir funciones de tejidos vivos y órganos en el cuerpo. Por tanto, es esencial entender las relaciones existentes entre las propiedades, funciones y estructuras de los materiales biológicos, dado que las propiedades requeridas de un material varían de acuerdo con la aplicación particular. Los biomateriales pueden ser: 1) inertes, ya que no desencadenan ninguna reacción en el huésped; 2) bioactivos, asegurando así un desempeño estable y duradero; y 3 ) biodegradables, que pueden ser degradados químicamente o descompuestos por factores naturales como las bacterias. Es de vital importancia que estos materiales biodegradables de uso médico no tengan riesgo de carcinogénesis, inmunogenicidad, teratogenicidad y toxicidad (7).

Los biomateriales más usados son las aleaciones a base de magnesio y polímeros sintéticos tales como: ácido poliláctico, ácido poliglicólico, policaprolactona, polidioxanona y poliláctico coglicólido (8-10). Los biomateriales hechos de aleaciones a base de magnesio son de alta biocompatibilidad y tienen la propiedad de disolverse en el cuerpo humano durante el proceso de degradación. Sin embargo, debido a su alta corrosión, la degradación ocurre antes de culminar su objetivo.

Los biomateriales de naturaleza polimérica tienen la ventaja de ser elásticos, de baja densidad y relativamente fáciles de fabricar. Los polímeros que se utilizan en los implementos médicos se degradan más lentamente que las aleaciones de magnesio y su degradación es hidrolítica. Este proceso de hidrólisis degrada los polímeros en moléculas de bajo peso molecular, las cuales pueden ser metabolizadas por el organismo.

El polímero ideal para ser utilizado en los stents biodegradables debe: 1) mantener una fuerza expansiva suficiente hasta que la estenosis quede resuelta; 2) ser no tóxicos; 3) que no induzca una respuesta inflamatoria en el tejido vecino; 4) metabolizarse en el cuerpo después de cumplir su función; 4) ser fácilmente procesado y que no deje trazas; y 5) ser fácil de esterilizar. Teniendo en cuenta estas características, las principales ventajas de un polímero sintético son: 1) adecuada biocompatibilidad; 2) la posibilidad de cambiar en composición y en las propiedades fisicomecánicas; 3) tener un coeficiente de baja fricción; 4) facilidad de ser procesado y viabilidad; 5) alternativa de cambio químico y físico en la superficie; y 6) habilidad de inmovilizar células o biomoléculas dentro de ellos o en su superficie (esta última característica llevó al descubrimiento de los stents con liberación de fármacos, como lo son algunos stents coronarios). Los polímeros biodegradables usados para la liberación de fármacos representan un gran avance tecnológico y se vienen utilizando principalmente en el sistema vascular con el uso de stents coronarios (11).

La necesidad generada por el desarrollo de fármacos que no pueden ser administrados por las vías tradicionales, intramuscular, subcutánea o endovenosa, y la frecuente conveniencia de suministrar un fármaco de manera localizada y controlada en el lugar donde debe ejercer su acción, han promovido un área de investigación y desarrollo de biomateriales dentro del campo de la farmacia. Por ejemplo, en la elaboración de dispositivos que incorporan un fármaco en una matriz bioabsorbible, la liberación y consiguiente disponibilidad del fármaco está determinada por la velocidad con que se degrada el polímero que la contiene (12). Los stents coronarios son un gran ejemplo. Se han implementado diferentes compuestos en la matriz del stent biodegradable, con el objeto de evitar la reacción intimal, tales como el tacrolimus, el sirolimus, el paclitaxel, la aspirina, la dexametasona $(13,14)$ e incluso material radioactivo para prevenir la reestenosis e inflamación (15). Hasta la fecha, no hay ninguna aplicación similar en el área gastroenterológica en humanos (figura 1).

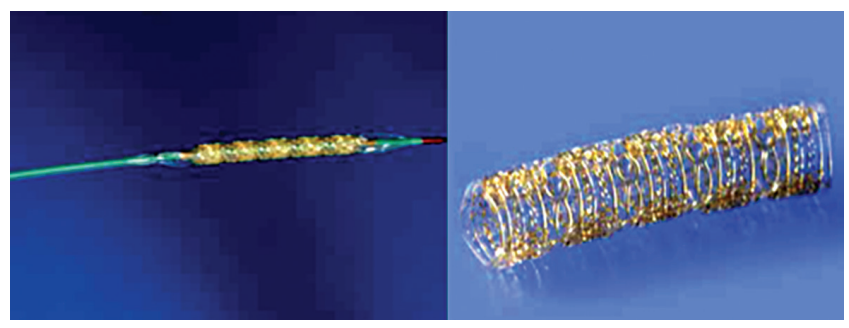

Figura 1. Stent coronario bioabsorbible y revestido con medicamento.

El mecanismo de acción de los polímeros varía según sean o no biodegradables. Aunque toda la materia se degrada con el paso del tiempo, el término biodegradable aplicado a los polímeros implica que este se elimina en un corto período. Los poliésteres son los polímeros sintéticos biodegradables de uso comercial que tienen mayor aplicación en el campo médico. Se caracterizan por la presencia de enlaces éster en la cadena principal, lo cual permite que sean degradados hidrolíticamente. Los procesos de hidrólisis degradan los polímeros en moléculas de bajo peso molecular que pueden ser metabolizadas por el organismo. El medio fisiológico humano reúne las condiciones apropiadas para que estos procesos se produzcan en condiciones normales a un $\mathrm{pH}$ entre 7 y 7,4 (16).

Como se describió, los stents biodegradables son hechos de varios polímeros sintéticos tales como ácido poliláctico, 
poliglicolato o ácido poliglicólico, poli-L- láctico (figura 2) y copolímeros de ácido poliláctico-coglicólico y polidioxanona (figura 3) (17). Los stents biodegradables han sido utilizados en el tratamiento de estenosis benignas refractarias tales como ureterales, uretrales, traqueales, biliares, pancreáticas, intestino delgado, colon y estenosis esofágicas (18).

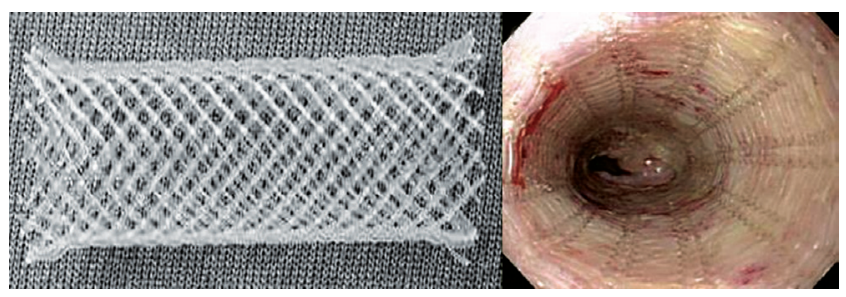

Figura 2. Stent biodegradable de ácido poli-L-láctico (PLLA).

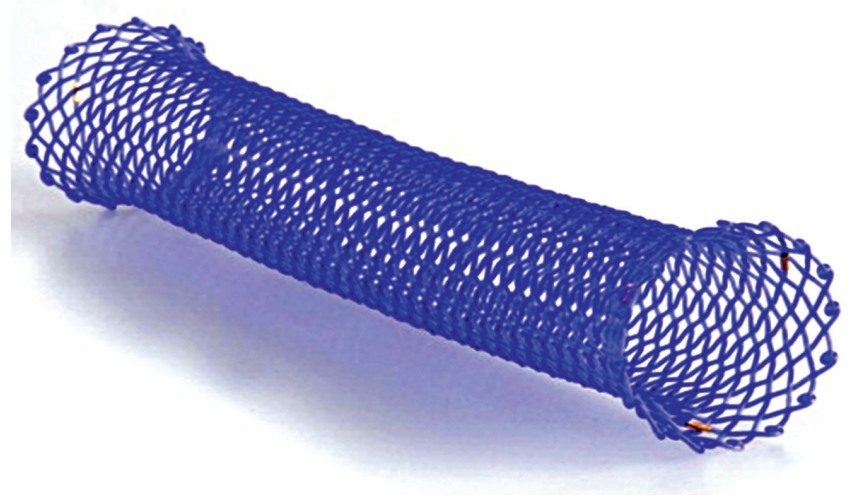

Figura 3. Stent biodegradable de polidioxanona.

\section{EXPERIENCIA CLÍNICA CON LOS STENTS GASTROINTESTINALES Y PANCREATOBILIARES BIODEGRADABLES}

La idea de utilizar stents biodegradables no es nueva; ya entre 1991 y 1992 había prototipos experimentales. No obstante, solo en los últimos años se dispuso de modelos que superaron la fase experimental en animales y comenzaron a ser tan seguros como para ser probados en seres humanos.

En 1993, Kemppainen publicó el primer estudio de stents biodegradables, en el que utilizó un modelo experimental de estenosis uretral en conejos tratados con un stent biodegradable hecho de ácido poli-L-láctico. En este estudio, los autores concluyeron que este tipo de stent tenía un gran futuro para prevenir la reestenosis de las estrecheces uretrales (19).

En el tracto gastrointestinal los primeros stents biodegradables fueron hechos de ácido poliláctico y desarrollados por Goldin (20), quien reporto la experiencia con 5 pacientes que tenían estenosis esofágica benigna refractaria al tratamiento endoscópico (figura 4). Los autores concluyeron que este prototipo de stent no fue capaz de mantener una fuerza radial significativa y sostenida por más de 3 semanas. El stent se desintegró en un lapso de 6 semanas después de la colocación e indujo la obstrucción del lumen esofágico. Estos hallazgos fueron confirmados en otro estudio realizado por Fry \& Fleisher (21).

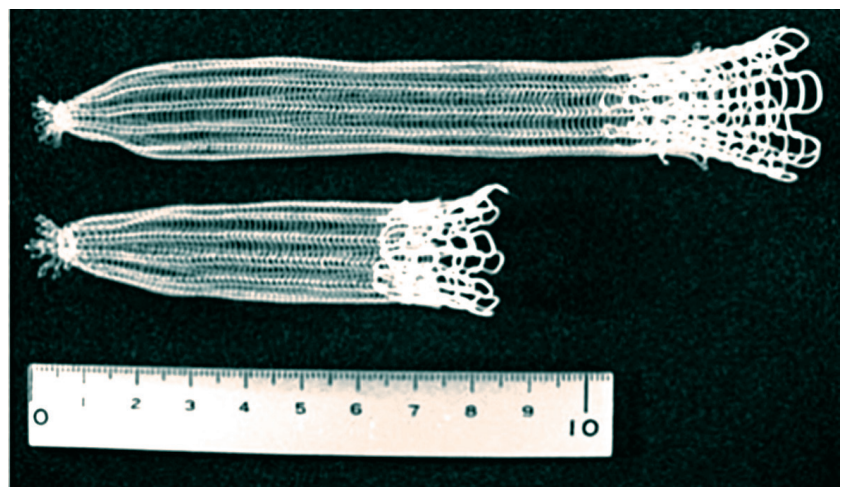

Figura 4. Stent biodegradable de ácido poli-L-láctico.

Los stents biodegradables hechos de polidioxanona han mejorado los resultados ostensiblemente (figura 5).

Este tipo de stent tiene una mayor integridad y conserva una fuerza radial que es mantenida durante 6 a 8 semanas después de la colocación, como lo pudo demostrar Rejchrt (22). En este estudio piloto de tres pacientes con estenosis benigna del intestino delgado y del colon, los autores confirmaron que la degradación y fragmentación del stent ocurre entre las 11-12 semana después de colocado el stent. Parece ser que la degradación del stent es dependiente del $\mathrm{pH}$ y es más rápida la degradación cuando el $\mathrm{pH}$ es más bajo. Es posible que estas observaciones preliminares donde se presentó una dilatación prolongada antes de la degradación y absorción del stent puedan representar una solución para pacientes con estenosis benignas refractarias a la dilatación convencional. Este nuevo tipo de stent biodegradable permite una dilatación radial constante que es similar a la que se logra con los stents metálicos expandibles, con la salvedad que el stent biodegradable no necesita ser removido. El stent biodegradable de polidioxanona puede ser una alternativa de tratamiento para las estenosis benignas refractarias del tracto digestivo.

Parviainen manejó dos anastomosis pancreatoyeyunales en forma profiláctica con stents biodegradables de polilactida. Ninguno de estos pacientes desarrolló complicaciones en el posoperatorio. Dichos stents se degradan más fácilmente en el medio pancreático que en el biliar (23).

\section{Esófago}

La colocación de stents biodegradables es una alternativa para el tratamiento de las estenosis esofágicas benignas 

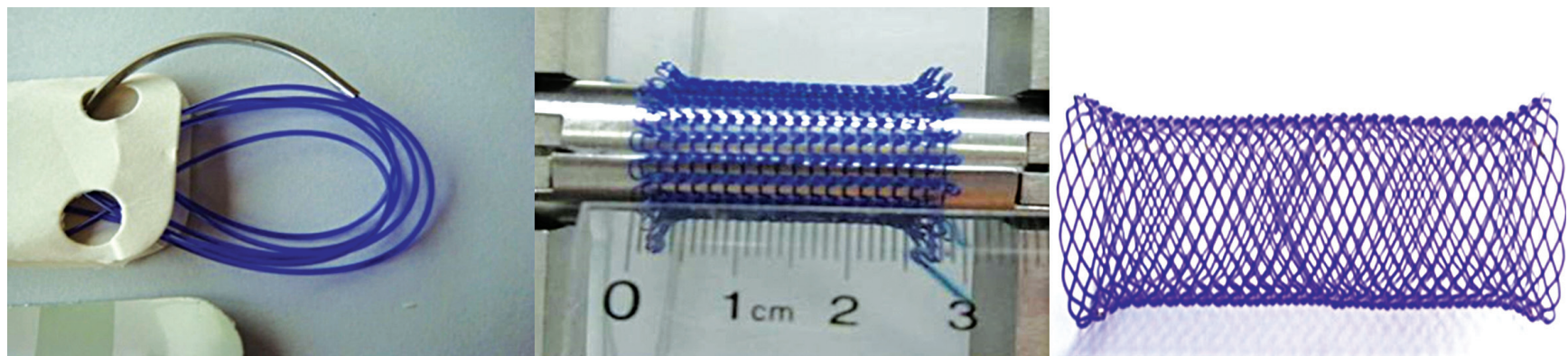

Figura 5. Stent biodegradable de polidioxanona en sus fases de fabricación.

refractarias al tratamiento convencional con dilatación. También podría ser una alternativa a considerar en pacientes con acalasia. Dichas estenosis esofágicas generalmente están relacionadas con el reflujo gastroesofágico, la ingestión de cáusticos, el posoperatorio de cirugía esofágica, y son secundarias a la radiación (18). El tratamiento convencional de estas estenosis es con dilataciones utilizando los dilatadores tipo bujía o con balones hidrostáticos. Este tipo de tratamiento está asociado con una mejoría clínica inmediata de la disfagia en un $80 \%-90 \%$ de los casos. Sin embargo, un $30 \%$ a $60 \%$ de las estenosis benignas puede recurrir a corto y largo plazo. Este tipo de pacientes con estenosis benignas recurrentes y recalcitrantes al tratamiento convencional son los que se pueden beneficiar de un tratamiento alternativo y los stents biodegradables fueron desarrollados para ser considerados en este grupo especial de pacientes (24). Saito reportó los resultados de dos series de pacientes los cuales fueron tratados con stents hechos de ácido poliláctico (figura 6). El 77\% de estos stents migró en un período de 10 a 21 días después de colocados. Sin embargo, se observó un éxito clínico en todos los pacientes por un período de 7 meses a 2 años (10).

En el 2007 se comenzó a utilizar el stent de ELLA (ELLA-Cs, Hradec Kralove, República Checa). Dicho stent biodegradable es hecho del polímero polidioxanona y es en la actualidad el único stent biodegradable utilizado en el tracto digestivo. El stent está ensamblado en un introductor $28 \mathrm{~F}$ y viene en diferentes tamaños entre 18 y $25 \mathrm{~mm}$. Es manufacturado para uso comercial y está hecho de polidioxanona, que es un polímero biodegradable semicristalino y se degrada por hidrólisis. Los productos de biodegradación no son tóxicos. El stent es radiotransparente y tiene marcas radiopacas en el extremo proximal y distal (figura 7) (25).

El estudio publicado por Rejchrt (25) en 2009 con el stent de polidioxanona demostró que la fuerza radial e integridad del stent fueron mantenidas de una forma continua al menos por 6 semanas después de la colocación. Esto es mucho mejor que la experiencia publicada anteriormente con otros stents biodegradables. El $60 \%$ de la degradación del stent ocurre entre las semanas 7 y 9 después de colocado y un $90 \%$ de degradación a las 9 semanas. Se recomienda tratamiento supresor del ácido ya que se ha demostrado que la degradación de este tipo de stent es más rápida con la exposición al ácido. El tiempo promedio de degradación de este tipo de stent ocurre entre las 11 y 12 semanas (figura 8).

Se ha publicado en varios estudios la experiencia con los stents biodegradables de polidioxanona SX-ELLA en el tratamiento de pacientes con estenosis benignas refractarias del esófago $(16,18,25-31)$ así como en la acalasia (32). $\mathrm{El}$ éxito técnico, la respuesta clínica y las complicaciones

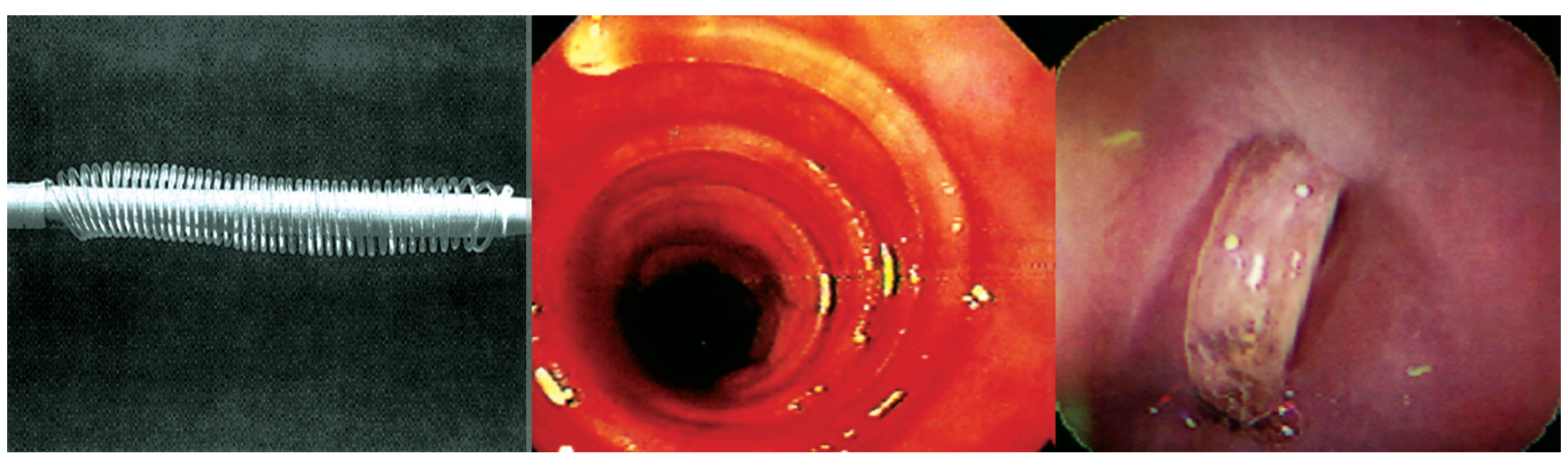

Figura 6. Stent biodegradable de ácido poliláctico. 

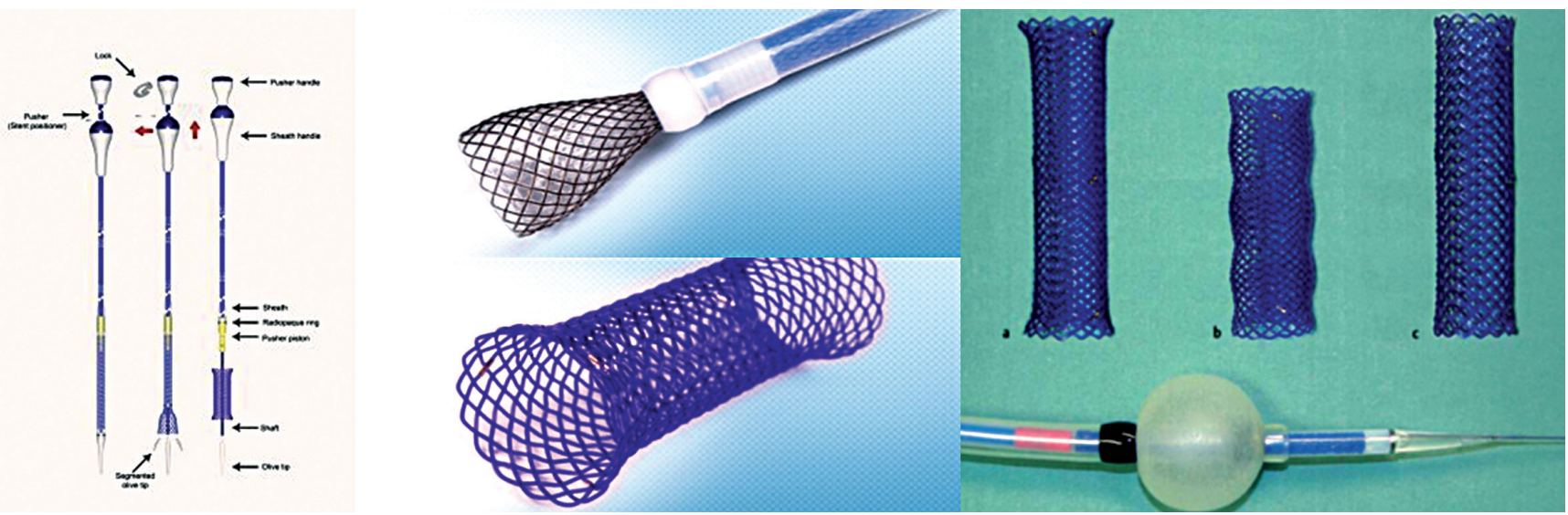

Figura 7. Stent biodegradable de polidioxanona por ELLA, República Checa.
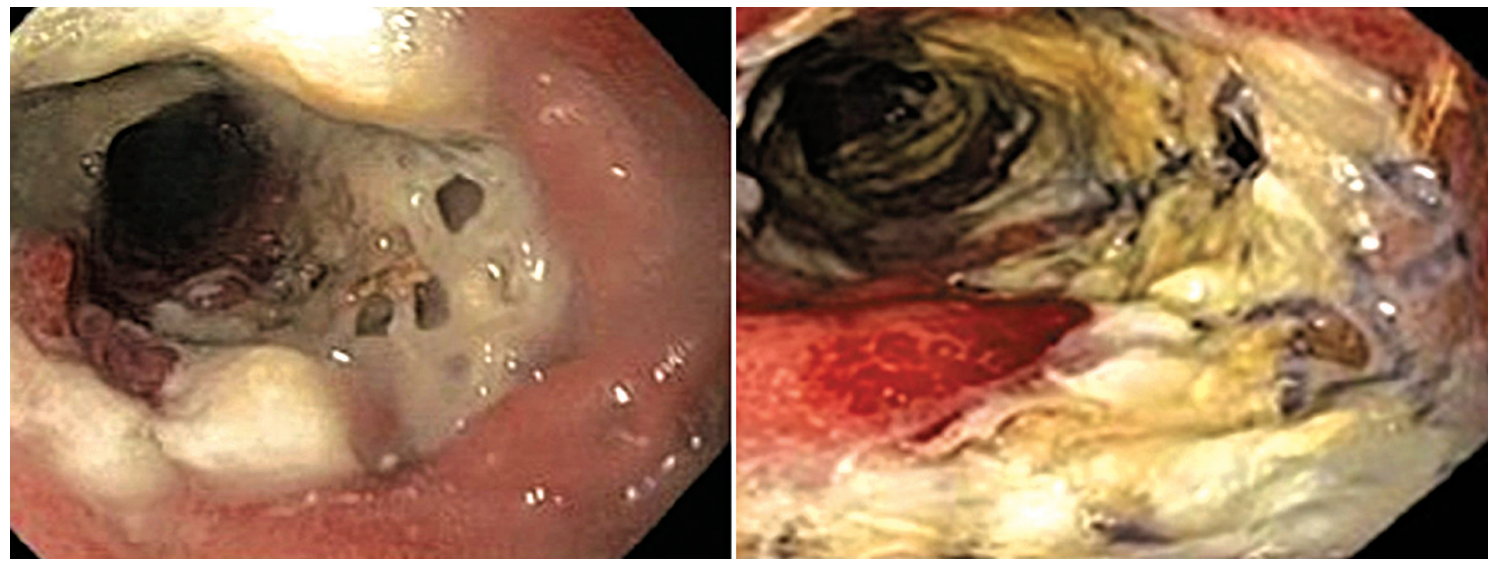

Figura 8. Imágenes endoscópicas del stent ELLA durante el proceso de biodegradación.

varían de acuerdo con el estudio. La colocación del stent no fue un problema para todos los casos reportados. El éxito clínico varía desde un $0 \%$ hasta un $100 \%$, con un promedio del 40\% (33-35). Repici (31) publicó en el 2010 un estudio prospectivo de dos centros europeos. En este estudio se utilizaron stents biodegradables en 21 pacientes con estenosis esofágicas refractarias al tratamiento endoscópico. Todos los pacientes fueron tratados con el stent ELLA (ELLA-CS, Hradec Kralove, República Checa) La colocación de los stents fue exitosa en el 100\% de los casos. La migración del stent ocurrió en solo 2 pacientes después de varias semanas de la colocación. La biodegradación del stent ocurrió en 3 a 6 meses. La endoscopia de seguimiento a los 3 meses mostró que todos los stents estaban fragmentados y la endoscopia de seguimiento a los 6 meses demostró que todos los stents se habían disuelto. Al final del seguimiento, 9 pacientes de 20 (45\%) tuvieron resolución de la disfagia. Para tener en consideración, la frecuencia de dilataciones endoscópicas fue menor en los pacientes en quienes falló el tratamiento con el stent. La serie con el mayor seguimiento a largo plazo con el uso de este tipo de stent fue reportada por Hirdes. En esta serie de 28 pacientes se alcanzó un éxito clínico del 25\% (28).

Los stents biodegradables teóricamente serían el tratamiento ideal para las estenosis benignas refractarias al tratamiento endoscópico. Dichos stents pueden proporcionar una permeabilidad temporal conjuntamente con una remodelación de la zona de estenosis. La eficacia temporal de los stents biodegradables parte de un concepto simple en el cual una estenosis que necesita dilatación repetitiva responderá a una dilatación prolongada con el uso de una endoprótesis que se dejará in situ por varias semanas o meses. El principio fundamental es obtener una remodelación de la estenosis fibrótica utilizando un stent hecho de materiales altamente biocompatibles que no induzcan un daño mecánico o irritativo que pueda resultar en crecimiento anormal del tejido de granulación o en la formación de una nueva estenosis o de un tracto fistuloso.

Tres tipos diferentes de stents expandibles se han utilizado para el tratamiento de las estenosis esofágicas refractarias, 
tales como el stent autoexpandible de plástico, los stents metálicos cubiertos autoexpandibles y los stents biodegradables. Hasta la fecha, solo se ha publicado un solo estudio que comparó la eficacia de estos tres tipos de stents. Canena (36) demostró que la colocación temporal de un stent metálico autoexpandible o un stent biodegradable tiene una utilidad similar en el tratamiento de las estenosis esofágicas refractarias con resolución de la disfagia a largo plazo, de un $40 \%$ y un $30 \%$, respectivamente. La colocación de los stents de plástico autoexpandibles estuvo asociada con el menor éxito clínico de los tres, de solo un $10 \%$. Dichos stents de plástico autoexpandibles también estuvieron asociados con una incidencia mayor de migración y reintervención. La migración con los stents metálicos expandibles fue del $30 \%$ comparado con los stents biodegradables, del 20\%. La implementación de dilatación con balón del stent biodegradable después de la colocación disminuyó la incidencia de migración.

El dolor torácico fue la complicación más frecuente que se ha reportado en los diferentes estudios. La hiperplasia de tejido ocurre en conjunto con la degradación del stent. Casos de hiperplasia severa con recurrencia de disfagia han sido reportados (29, 32-35). Para solucionar este problema, se ha reportado tratamiento con argón plasma $(29,32)$ o dilatación con balón (35). Otras complicaciones potenciales de los stents biodegradables son el colapso del stent en el lumen esofágico y la fístula traqueoesofágica (37).

Se puede concluir que después de revisar las series publicadas hasta la fecha, parece que un subgrupo de pacientes con este tipo de estenosis esofágica refractaria al tratamiento endoscópico está destinado a un tratamiento prolongado con dilataciones, lo que incrementa el riesgo de complicaciones, los costos inherentes al tratamiento y reduce significativamente la calidad de vida. Este subgrupo de pacientes serían candidatos al tratamiento con stents biodegradables. Los estudios que se han publicado hasta la fecha tienen limitaciones en el diseño; sin embargo, han demostrado que los stents biodegradables son efectivos, seguros y representan una alternativa muy atractiva en este subgrupo de pacientes.

\section{Intestino delgado y colon}

Los stents biodegradables pueden ser una opción terapéutica promisoria en pacientes con estenosis del intestino delgado y del colon. La primera descripción de un stent biodegradable en esta ubicación se le atribuye al grupo checo de Rejchrt (22). Las estenosis posquirúrgicas, principalmente después de cirugía de colon y recto, son las más frecuentes y ocurren entre el 1,5\% y el $8 \%$ de los pacientes. Las estenosis también pueden ocurrir después de la resección de cáncer de colon (38-41) en casos de resección de fístulas colónicas (35) y también en casos de enfermedad de Crohn con estenosis $(22,42)$. La colocación a nivel intestinal es posible y es relativamente simple excepto en casos de estenosis proximales, cuando hay una deformidad o angulación marcada. El introductor tiene una longitud aproximada de 75 $\mathrm{cm}$, lo cual es una limitante. En la mayoría de los casos, es difícil colocar un stent a más de $30 \mathrm{~cm}$ del ano. Para esto, se ha sugerido el uso de un sobretubo (22). La migración del stent es una de las grandes limitantes y es la razón principal de falla en la respuesta clínica. Se han utilizado diferentes técnicas para evitar la migración. Hasta la fecha no se ha documentado hiperplasia de la mucosa en casos de estenosis intestinales tratadas con stents biodegradables (figura 9).

Se puede decir que los stents biodegradables son una nueva opción para el tratamiento de estenosis del intes-
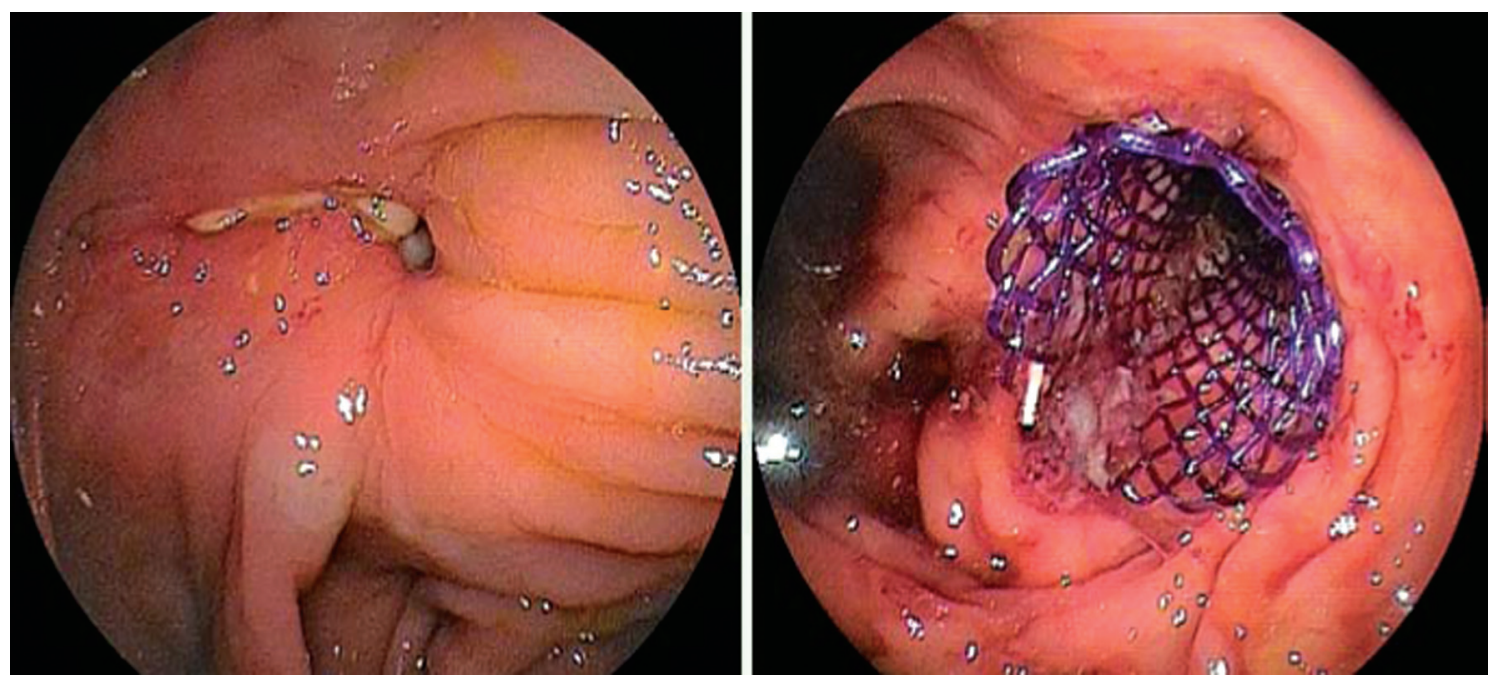

Figura 9. Imágenes endoscópicas de estenosis ileocolónica antes y después de la colocación del stent biodegradable. 
tino delgado y del colon posquirúrgico y secundario a enfermedad de Crohn. El uso de este tipo de stents disminuye la necesidad de repetir endoscopias y dilataciones. Consecuentemente disminuye el riesgo de perforaciones y procedimientos quirúrgicos. La alta incidencia de migración temprana se puede solucionar con mejorías en el diseño del stent. Se necesitan más estudios para determinar la eficacia y seguridad de estos stents a largo plazo.

\section{Tracto biliar y pancreático}

La endoscopia es el tratamiento de elección para las estenosis biliares benignas. El papel de los stents biodegradables en la práctica clínica no está bien establecido y es una de las áreas de investigación actual en diferentes modelos animales $(43,44)$. Las causas de estenosis biliares son múltiples pero las dos más comunes son las estenosis posquirúrgicas y las estenosis secundarias a pancreatitis crónica (45). Los stents disponibles actualmente en el mercado para los conductos biliares y el conducto pancreático son de plástico o metálicos. Dichos stents tienen sus limitaciones aun en los estudios animales $(46)$. Laukkarinen $(47,48)$ publicó diferentes estudios con modelos animales en los que usó stents biodegradables en los conductos biliares y en el conducto pancreático. Este grupo de autores ha investigado la degradación, permeabilidad y toxicidad de los stents biodegradables hechos de ácido poliláctico. Estos stents han sido usados en cerdos y después de 6 meses de colocados no se han observado cambios histológicos o anatómicos.

Petrtyl (49) y Mauri (50) han publicado su experiencia con el uso de stents biodegradables de polidioxanona colocados por vía percutánea en el conducto biliar. En estos dos estudios se trataron 12 pacientes con estenosis biliar posquirúrgica y se demostró un buen éxito clínico a 2 años de seguimiento.

\section{CONCLUSIONES}

Aunque no existen en el mercado nacional los stents biodegradables, sus costos en el momento son prohibitivos. La experiencia acumulada hasta la fecha en otras latitudes, en donde utilizó el único stent biodegradable de uso endoscópico y hecho del polímero de polidioxanona, ha demostrado resultados promisorios. La revisión de la literatura permite decir que los stents biodegradables son una nueva opción para el tratamiento de estenosis refractarias del esófago, estenosis biliar, del intestino delgado y del colon, como también estenosis secundarias a enfermedad de Crohn. El uso de este tipo de stents disminuye la necesidad de repetir endoscopias y dilataciones. Consecuentemente disminuye el riesgo de perforaciones y procedimientos quirúrgicos. La alta incidencia de migración temprana se puede solucionar con mejorías en el diseño del stent. Se necesitan más estudios para determinar la eficacia y seguridad de estos stents a largo plazo. Se debe mejorar la pérdida de la fuerza radial con el tiempo debido a la degradación.

Sin duda se necesitan estudios donde se pueda comparar el uso de estos stents biodegradables versus stents expandibles totalmente cubiertos. Los seguimientos de los estudios deben ser a largo plazo para evaluar la remisión de los síntomas con el uso de estos stents. También sería de gran importancia evaluar el uso de estos stents biodegradables en pacientes con cáncer esofágico o disfagia maligna antes de la quimioterapia o la radioterapia. Por último, el stent ideal para las estenosis benignas debería ser de diámetro amplio, alta expansión, alta flexibilidad, que mantenga la integridad luminal, que no cause hiperplasia epitelial o daño del tejido y que no se necesite repetir la endoscopia para la extracción del stent. Es muy probable que los stents biodegradables sean el stent ideal para lograr estas metas en un futuro.

\section{REFERENCIAS}

1. Tringali A, Blero D, Boskoski I, et al. Difficult removal of fully covered self expandable metal stents (SEMS) for benign biliary strictures: The "SEMS in SEMS" technique. Dig Liver Dis. 2014;46(6):568-71.

2. Sharaiha RZ, Kim KJ, Singh VK, et al. Endoscopic stenting for benign upper gastrointestinal strictures and leaks. Surg Endosc. 2014;28:178-84.

3. Almadi MA, Azzam N, Alharbi O, Mohammed AH, Sadaf $\mathrm{N}$, Aljebreen AM. Complications and survival in patients undergoing colonic stenting for malignant obstruction. World J Gastroenterol. 2013;19:7138-45.

4. van Boeckel PG, Dua KS, Weusten BL, et al. Fully covered self-expandable metal stents (SEMS), partially covered SEMS and self-expandable plastic stents for the treatment of benign esophageal ruptures and anastomotic leaks. BMC Gastroenterology. 2012;12:19.

5. Gutierrez-Salmean G, Pelaez-Luna M, Gonzalez-Galeote E, Lozoya-Gonzalez D, Fuchs-Tarlovsky V, Farca-Belsaguy A. Outcomes of temporary self-expanding plastic stents (SEPS) use for gastrointestinal leaks. A case series. Rev Gastroenterol Méx. 2009;74:181-6.

6. Committee AT, Tokar JL, Banerjee S, et al. Drug-eluting/ biodegradable stents. Gastrointest Endosc. 2011;74:954-8.

7. Lorenzo-Zuniga V, Moreno-de-Vega V, Marin I, Boix J. Biodegradable stents in gastrointestinal endoscopy. World J Gastroenter. 2014;20:2212-7.

8. Guo Q, Knight PT, Mather PT. Tailored drug release from biodegradable stent coatings based on hybrid polyurethanes. J Control Release. 2009;137:224-33.

9. Hermawan H, Dube D, Mantovani D. Degradable metallic biomaterials: design and development of Fe-Mn alloys for stents. J Biomed Mater Res A. 2010;93:1-11.

10. Saito Y, Tanaka T, Andoh A, et al. Usefulness of biodegradable stents constructed of poly-l-lactic acid monofila- 
ments in patients with benign esophageal stenosis. World J Gastroenter. 2007;13:3977-80.

11. Neamtu I, Chiriac AP, Diaconu A, Nita LE, Balan V, Nistor MT. Current concepts on cardiovascular stent devices. Mini Rev Med Chem. 2014;14(6):505-36.

12. Austin AS, Khan Z, Cole AT, Freeman JG. Placement of esophageal self-expanding metallic stents without fluoroscopy. Gastrointest Endosc. 2001;54:357-9.

13. Han Y, Jing Q, Xu B, et al. Safety and efficacy of biodegradable polymer-coated sirolimus-eluting stents in «real-world» practice: 18-month clinical and 9-month angiographic outcomes. JACC. 2009;2:303-9.

14. Onuma Y, Serruys P, den Heijer P, et al. MAHOROBA, firstin-man study: 6-month results of a biodegradable polymer sustained release tacrolimus-eluting stent in de novo coronary stenoses. Eur Heart J. 2009;30:1477-85.

15. Wykrzykowska JJ, Onuma Y, Serruys PW. Advances in stent drug delivery: the future is in bioabsorbable stents. Expert Opin Drug Deliv. 2009;6:113-26.

16. Basha J, Appasani S, Vaiphei K, Gupta V, Singh K, Kochhar R. Biodegradable stents: truly biodegradable with good tissue harmony. Endoscopy. 2013;45(Suppl 2):E116-7.

17. Athanasiou KA, Niederauer GG, Agrawal CM. Sterilization, toxicity, biocompatibility and clinical applications of polylactic acid/polyglycolic acid copolymers. Biomaterials. 1996; 17:93-102.

18. Griffiths EA, Gregory CJ, Pursnani KG, Ward JB, Stockwell RC. The use of biodegradable (SX-ELLA) oesophageal stents to treat dysphagia due to benign and malignant oesophageal disease. Surg Endosc. 2012;26:2367-75.

19. Kemppainen E, Talja M, Riihela M, Pohjonen T, Tormala P, Alfthan O. A bioresorbable urethral stent. An experimental study. Urol Res. 1993;21:235-8.

20. Goldin E, Fiorini A, Ratan Y, et al. A new biodegradable and self-expandable stent for benign esophageal strictures. Gastrointest Endosc. 1996;43:294.

21. Fry SW, Fleischer DE. Management of a refractory benign esophageal stricture with a new biodegradable stent. Gastrointest Endosc. 1997;45:179-82.

22. Rejchrt S, Kopacova M, Brozik J, Bures J. Biodegradable stents for the treatment of benign stenoses of the small and large intestines. Endoscopy. 2011;43:911-7.

23. Parviainen M, Sand J, Harmoinen A, et al. A new biodegradable stent for the pancreaticojejunal anastomosis after pancreaticoduodenal resection: in vitro examination and pilot experiences in humans. Pancreas. 2000;21:14-21.

24. Tanaka T, Takahashi M, Nitta N, et al. Newly developed biodegradable stents for benign gastrointestinal tract stenoses: a preliminary clinical trial. Digestion. 2006;74:199-205.

25. Rejchrt S, Kopacova M, Bartova J, Bures J. Intestinal biodegradable stents. initial experience in the czech republic. Folia Gastroenterol Hepatol. 2009; 7:7.

26. Vandenplas Y, Hauser B, Devreker T, Urbain D, Reynaert H. A biodegradable esophageal stent in the treatment of a corrosive esophageal stenosis in a child. J Pediatr Gastroenterol Nutr. 2009;49:254-7.
27. van Hooft JE, van Berge Henegouwen MI, Rauws EA, Bergman JJ, Busch OR, Fockens P. Endoscopic treatment of benign anastomotic esophagogastric strictures with a biodegradable stent. Gastrointest Endosc. 2011;73:1043-7.

28. Hirdes MM, Siersema PD, van Boeckel PG, Vleggaar FP. Single and sequential biodegradable stent placement for refractory benign esophageal strictures: a prospective follow-up study. Endoscopy. 2012;44:649-54.

29. Karakan T, Utku OG, Dorukoz O, et al. Biodegradable stents for caustic esophageal strictures: a new therapeutic approach. Dis Esophagus. 2013;26:319-22.

30. Stivaros SM, Williams LR, Senger C, Wilbraham L, Laasch HU. Woven polydioxanone biodegradable stents: a new treatment option for benign and malignant oesophageal strictures. Eur Radiol. 2010;20:1069-72.

31. Repici A, Vleggaar FP, Hassan C, et al. Efficacy and safety of biodegradable stents for refractory benign esophageal strictures: the BEST (Biodegradable Esophageal Stent) study. Gastrointest Endosc. 2010;72:927-34.

32. Hair CS, Devonshire DA. Severe hyperplastic tissue stenosis of a novel biodegradable esophageal stent and subsequent successful management with high-pressure balloon dilation. Endoscopy. 2010;(42 Suppl 2):E132-3.

33. Orive-Calzada A, Alvarez-Rubio M, Romero-Izquierdo S, et al. Severe epithelial hyperplasia as a complication of a novel biodegradable stent. Endoscopy. 2009;41(Suppl 2):E137-8.

34. Fischer A, Bausch D, Baier P, Braun A, Richter-Schrag H. Risk of biodegradable stent-induced hypergranulation causing re-stenosis of a gastric conduit after esophageal resection. Endoscopy. 2012;44(Suppl 2):E125-6.

35. Dumoulin FL, Plassmann D. Tissue hyperplasia following placement of a biodegradable stent for a refractory esophageal stricture: treatment with argon plasma coagulation. Endoscopy. 2012;44(Suppl 2):E356-7.

36. Canena JM, Liberato MJ, Rio-Tinto RA, et al. A comparison of the temporary placement of 3 different self-expanding stents for the treatment of refractory benign esophageal strictures: a prospective multicentre study. BMC. 2012;12:70.

37. Jung GE, Sauer P, Schaible A. Tracheoesophageal fistula following implantation of a biodegradable stent for a refractory benign esophageal stricture. Endoscopy. 2010;42(Suppl 2):E338-9.

38. Repici A, Pagano N, Rando G, et al. A retrospective analysis of early and late outcome of biodegradable stent placement in the management of refractory anastomotic colorectal strictures. Surg Endosc. 2013;27:2487-91.

39. Perez Roldan F, Gonzalez Carro P, Villafanez Garcia MC, et al. Usefulness of biodegradable polydioxanone stents in the treatment of postsurgical colorectal strictures and fistulas. Endoscopy. 2012;44:297-300.

40. Toth E, Nielsen J, Nemeth A, et al. Treatment of a benign colorectal anastomotic stricture with a biodegradable stent. Endoscopy. 2011;43(Suppl 2):E252-3.

41. Janik V, Horak L, Hnanicek J, Malek J, Laasch HU. Biodegradable polydioxanone stents: a new option for 
therapy-resistant anastomotic strictures of the colon. Eur Radiol. 2011;21:1956-61.

42. Rodrigues C, Oliveira A, Santos L, Pires E, Deus J. Biodegradable stent for the treatment of a colonic stricture in Crohn's disease. World J Gastrointest Endosc. 2013;5:265-9.

43. Ginsberg G, Cope C, Shah J, et al. In vivo evaluation of a new bioabsorbable self-expanding biliary stent. Gastrointest Endosc. 2003;58:777-84.

44. Meng B, Wang J, Zhu N, Meng QY, Cui FZ, Xu YX. Study of biodegradable and self-expandable PLLA helical biliary stent in vivo and in vitro. J Mater Sci Mater Med. 2006;17:611-7.

45. Kaffes AJ, Liu K. Fully covered self-expandable metal stents for treatment of benign biliary strictures. Gastrointest Endosc. 2013;78:13-21.

46. Itoi T, Kasuya K, Abe Y, Isayama H. Endoscopic placement of a new short-term biodegradable pancreatic and biliary stent in an animal model: a preliminary feasibility study (with videos). J Hepatobiliary Pancreat Sci. 2011;18:463-7.

47. Lamsa T, Jin H, Mikkonen J, Laukkarinen J, Sand J, Nordback I. Biocompatibility of a new bioabsorbable radiopaque stent material (BaSO4 containing poly-L,D-lactide) in the rat pancreas. Pancreatology. 2006;6:301-5.

48. Laukkarinen JM, Sand JA, Chow P, et al. A novel biodegradable biliary stent in the normal duct hepaticojejunal anastomosis: an 18-month follow-up in a large animal model. J Gastrointest Surgery. 2007;11:750-7.

49. Petrtyl J, Bruha R, Horak L, Zadorova Z, Dosedel J, Laasch HU. Management of benign intrahepatic bile duct strictures: initial experience with polydioxanone biodegradable stents. Endoscopy. 2010;42(Suppl 2):E89-90.

50. Mauri G, Michelozzi C, Melchiorre F, et al. Biodegradable biliary stent implantation in the treatment of benign bilioplastic-refractory biliary strictures: preliminary experience. Eur Radiol. 2013;23:3304-10. 